\title{
EFFECT OF ULTRASOUND-ACTIVATED MICROBUBBLES ON THE CELL ELECTROPHYSIOLOGICAL PROPERTIES.
}

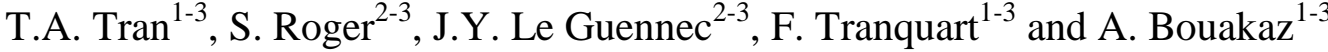 \\ 1. Inserm U619; 37000 Tours, France \\ 2. Inserm E0211; 37000 Tours, France \\ 3. Université François Rabelais; 37000 Tours, France
}

Running title: Ultrasound, microbubble and cell interaction.

Corresponding author:

Ayache Bouakaz, PhD

Inserm U619, B1A, Hôpital Bretonneau

2 bd Tonnellé, 37044 Tours Cedex 9, France

Tel: +33 (2) 47479748

Fax: +33 (2) 47479767

Email: bouakaz@med.univ-tours.fr 


\begin{abstract}
New clinical applications of ultrasound contrast microbubbles extend beyond imaging and diagnosis towards therapeutic applications. Cell membrane permeability and the uptake of substances have been shown to be enhanced by microbubbles under ultrasound stimulation. However, the mechanisms of action of ultrasound-activated microbubbles are still unknown. The aim of our study was to examine how microbubbles and ultrasound interact with cells in an attempt to understand the sonoporation mechanism. The ruptured-patch- clamp whole cell technique was used to measure membrane potential variations of a single cell. Sonovue microbubbles and mammary breast cancer cell line MDA-MB-231 were used. Ultrasound was applied using single element transducers of $1 \mathrm{MHz}$. Microbubbles and cells were simultaneously video-monitored during ultrasound exposure. Our results showed that during sonoporation, a marked cell membrane hyperpolarization occurs ( $\mathrm{n}=6$ cells) at negative pressures above $150 \mathrm{kPa}$, indicating the activation of specific ion channels while the cell and the microbubbles remain viable. The hyperpolarization was sustained as long as the microbubbles are in a direct contact with the cell and the ultrasound waves are transmitted. Smaller acoustic amplitudes induced only mild hyperpolarization while shutting off the ultrasound brings the cell membrane potential to its resting value. However ultrasound alone did not affect the cell membrane potential. A similar hyperpolarization of the cell membrane was observed when a mechanical pressure was applied on the cell through a glass probe. In conclusion, the results demonstrate that microbubbles' oscillations under ultrasound activation entail modifications of the electrophysiological cell activities, by triggering the modulation of ionic transports through the plasmic cell membrane. However, only cells in a direct contact with the microbubbles are impacted. The involved mechanisms are likely related to activation of specific channels sensitive to mechanical stresses (stretch-activated channels) and possibly non-specific ion channels.
\end{abstract}

Keywords: contrast, microbubbles, ultrasound, sonoporation, hyperpolarization, ionic channels. 


\section{Introduction:}

Ultrasound contrast agents (UCA) are now being used in various applications such as radiology and cardiology (Goldberg et al. 2001). Contrast agents of the first generation were composed of free air bubbles while newer generations consist of encapsulated microbubbles of gas that are sufficiently stable to pass into the systemic circulation following injection into a peripheral vein. The bubbles contain either air or a low solubility gas to lower the diffusion rate and to increase their lifetime in the blood. Most of these agents are intended to enhance the echo from the blood pool. The enhancement of the backscattered echo renders blood more detectable in B-mode imaging and Doppler mode. More stable contrast microbubbles allowed the development of sophisticated ultrasound imaging methods and by that the extension of the clinical applications of contrast echography.

Besides new contrast imaging applications, contrast microbubbles give real opportunities for therapeutic applications with ultrasound. Indeed, the future clinical applications of microbubbles are expanding towards therapy where they are exploited as gene and drug delivery systems (Unger et al. 2001; Unger et al. 2002). Various research groups have shown that microbubbles under optimal ultrasound scanning conditions increase the permeability of cell membrane to external substances (drugs or genes) and enhance by that their uptake in a sonoporation process (Christiansen et al. 2003; Taniyama et al. 2002). Recent studies have also shown pores formation (Tachibana et al. 1999), dynamic vesicle deformation and lysis due to microstreaming and strain induced by low-amplitude bubble oscillations (Marmottant and Hilgenfeldt 2003). In a recent study by Prentice et al (Prentice et al. 2005), using holographic optical trapping, they showed that ultrasound activated microbubbles in proximity to naked coverslips undergo microjet formation directed towards the surface. Interrogating Optison microbubbles at acoustic pressures up to $1.3 \mathrm{MPa}$, their results demonstrated the development of a micrometer width filament indicating the generation of 
microjets which presumably are responsible for cell permeation. Although high acoustic pressures were used, sonoporation can also occur at acoustic pressures within the low diagnostic range (van Wamel et al. 2004). In addition to being a sonoporation promoter, microbubbles offer the possibility to be loaded with drugs or genes inside the encapsulating shell. Conceptually, ultrasound-mediated destruction of microbubble carrier will provide selective and local release of the therapeutic compound in the targeted tissue. Despite the various progresses, the veritable mechanisms of interaction between ultrasound-bubbles and cells are still far from being understood. In addition, the effects by which ultrasound and gas bubbles augment cell membrane permeability are not elucidated due likely to the lack of methods for real-time monitoring of sonoporation at the cellular level. In a recent paper by Deng and co-workers (Deng et al. 2004), a voltage clamp technique was used where they explored the effects of ultrasound and Optison microbubbles on Xenopus oocytes, unfertilized egg cells. In their paper, the authors investigated the increase in membrane permeability through the measurement of inward current in voltage-clamp. While this paper presents a lot of interest, it does not describe the interactions between microbubbles and cell membrane. Also, the involvement of ionic channels activity cannot be ruled out.

To investigate the mechanisms involved in the sonoporation process at the cellular level, we used the patch clamp technique in ruptured whole-cell configuration. With this arrangement, we examined the response and the membrane permeabilization of single mammary cancer cells to oscillating Sonovue microbubbles in sonoporation conditions. The experimental setup permits the measurements of the membrane potential modification directly correlated with ionic exchange through the plasma membrane on the mammary cancer cells. 


\section{Materials and methods}

\section{Mammary cancer cells:}

Mammary cancer cells issued from MDA-MB-231 cell lines (American Type Culture Collection, Rockville, MD, USA) were used in this study. These cell lines were chosen since they represent a reference line for in-vitro studies of breast cancer mechanisms (Soule et al. 1973). The cells were cultured in a DMEM culture medium (Dulbecco's Modified Eagle's Medium, Cambrex, Belgium), supplemented with 5\% bovine serum, and then incubated at $37^{\circ} \mathrm{C}$ in the area satured by $\mathrm{H} 2 \mathrm{O}$ and $5 \% \mathrm{CO} 2$.

\section{Electrophysiology protocol and patch-clamp technique:}

To assess the effects of ultrasound and microbubbles on cell membrane, patch clamp technique was used in the experiment. Patch clamp is a technique able to visualize and to quantify micro and macro ion currents through the cell membrane. We used in our study the patch clamp in a "whole cell" configuration (Hamill et al. 1981; Neher and Sakmann 1976). The patch clamp setup rested on an inverted microscope (Eclipse TE300, Nikon, Champigny sur Marne, France) on an anti-vibrating table (TMC, MA, USA) as shown in figure 2. For electrophysiological analyses, cells were placed into 35-mm Petri dishes (Corning, NY, USA) at 3000 cells $/ \mathrm{cm}^{2}$. Before patch-clamping, after 2 incubating days, the growth medium DMEM (Dulbecco's Modified Eagle's Medium, Cambrex, Belgium) was washed and replaced with physiological saline solution (PSS: $140 \mathrm{mM} \mathrm{NaCl}, 4 \mathrm{mM} \mathrm{KCl}, 1 \mathrm{mM} \mathrm{MgCl}$, $0.33 \mathrm{mM} \mathrm{NaH} \mathrm{PO}_{4}, 10 \mathrm{mM}$ HEPES, $11.1 \mathrm{mM}$ Glucose, $\left.2 \mathrm{mM} \mathrm{CaCl} 2, \mathrm{pH}=7.4\right)$. Patch pipettes were prepared from non-heparinized haematocrit tubes (Vitrex, Paris, France) to a resistance of 3-5 M $\Omega$ with a heater puller (Sutter Instrument Co., CA, USA). A solution which composition is similar to the physiological intracellular medium was injected into the pipette (125 mM K-Glutamate, $0.37 \mathrm{mM} \mathrm{CaCl} 2,20 \mathrm{mM} \mathrm{KCl}, 1 \mathrm{mM} \mathrm{MgCl} 2,1 \mathrm{mM} \mathrm{MgATP}, 10 \mathrm{mM}$ 
HEPES, $1 \mathrm{mM}$ EGTA, pH=7.2). Membranes potentials were recorded under a particular current-clamp mode as no current was injected $(\mathrm{I}=0$ A) at room temperature using an Axopatch 200B patch clamp amplifier (Axon Instrument, Burlingame, CA, USA). Analogue signals were filtered at $5 \mathrm{kHz}$, using a five-pole lowpass Bessel filter, and sampled at $10 \mathrm{kHz}$ using a 1322A Digidata converter (Axon Instrument, Burlingame, CA, USA). PClamp software (v.9.2, Axon Instrument, Burlingame, CA, USA) was used for generation of voltage commands, acquisition and analysis of results. The cell under investigation was continuously perfused $(0.2 \mathrm{ml} / \mathrm{min})$ with PSS or test solutions using a peristaltic pump (Reglo Digital, Ismatec SA, Glattbrugg, Switzerland).

The cell was placed in a bath containing a physiological saline solution. In the first step, a glass micropipette with a tip of only a few micrometers in diameter was applied gently on the cell membrane to form a seal between the glass pipette and the membrane. The seal consists of creating an impermeable interaction between the plasma membrane and the glass probe (Fig. 1a). When the seal was realized, suction was then applied to the micropipette breaking a tiny section of the membrane allowing by that the solution inside the micropipette to mix up with the cytoplasm (Fig. 1b). The ionic composition was similar to the physiologic intracellular medium. When equilibrium was reached the ion activity could be recorded by measuring macroscopic currents, whole cell current and membrane potential (Fig. 1c).

During the patch clamp experiments, a video recording of the patched cell was simultaneously carried out using a standard video camera (Mintron MTV-7266PD, Taipei Hsien, Taiwan) connected to the microscope (Fig. 2).

Sonovue microbulles generously provided by Bracco Research Geneva were used in our experiments. Sonovue is composed of sulfur hexafluoride gas bubbles coated by a highly elastic phospholipid monolayer shell. The size distribution of Sonovue bubbles ranges from 1 to $12 \mu \mathrm{m}$ with a total number of $2 \times 10^{8} / \mathrm{ml}$ (Schneider et al. 1995). Sonovue was diluted to 
$1 / 250$ and the microbubbles were infused to the cells using a peristaltic pump at a rate of $1 \mathrm{ml}$ per minute.

To interrogate the bubbles and the patched cell, single element broadband transducer (Sofranel, Sartrouville France) was used operating at center frequencies of $1 \mathrm{MHz}$ and focused at $14 \mathrm{~mm}$. The selected transducer was mounted in a Plexiglas holder and positioned at an angle of $45^{\circ}$ from the targeted cells. Electrically gated signal was generated by an arbitrary waveform generator (Agilent technologies), and amplified using a linear power amplifier (Amplifier Research, Souderton, PA). The generated waveforms had negative pressure amplitudes up to $500 \mathrm{kPa}$, pulse lengths extending from 5 to 40 cycles, a repetition time of $100 \mu$ s and exposure times extending from 2 to 20 seconds. The acoustic pressures were measured separately using a needle hydrophone (Precision acoustics, UK). The patchclamp setup and ultrasound arrangement are shown in a schematic drawing in Fig. 2.

Growth and viability of cells were measured as a whole by the tetrazolium salt assay (Mosmann 1983). Cells were incubated at $37^{\circ} \mathrm{C}$ with the tetrazolium salt (3-[4.5dimethylthiazol-2-yl]-2.5-diphenyl tetrazolium bromide) and metabolically active cells reduced the dye to purple formazan. Formazan crystals were dissolved with DMSO. The absorbance was measured at $570 \mathrm{~nm}$. Cell proliferation was expressed as formazan $570 \mathrm{~nm}$ absorbance and not converted to cell numbers since there always was a control condition on each day of experiment. The cells were seeded into 6-well microplates at 3000 cells per well. After incubation for $24 \mathrm{~h}$, the cells were exposed to ultrasound and microbubbles and then reincubated. A concentration corresponding to approximately 10 microbubbles per cell was used. The experiments were carried out using the $1 \mathrm{MHz}$ transducer described earlier. Ultrasound exposure time was 1 min per culture dish. During insonification, the transducer was displaced to cover the whole culture dish. After $48 \mathrm{~h}$, the cells were washed with phosphate-buffered saline and $50 \mu \mathrm{l}$ of $5 \mathrm{mg} / \mathrm{ml}$ MTT solution in PBS were added to each 
well. The plates were incubated under cell culture conditions for $4 \mathrm{~h}$ and the formazan crystals were dissolved by adding $100 \mu$ dimethyl sulfoxide (DMSO) to each well. The absorptions were measured in triplicate at $570 \mathrm{~nm}$, with a background correction at $630 \mathrm{~nm}$, using a microplate ELISA reader. Results were recorded as percentage absorbance relative to untreated control cells. The cell viability was evaluated using the same ultrasound parameters as those used in the patch clamp measurements.

\section{Results and discussions}

\section{Effects of US and microbubbles on electrophysiological properties of cells:}

Figure 3 shows a typical optical observation displaying an MDA-MB 231 cell with the micropipette patched to its membrane.

The results obtained from the patch clamp experiments showed that microbubbles alone or ultrasound alone did not affect the electrophysiological properties of the cell membrane. As shown in Fig. 4, the membrane potential of the cell exposed to waveforms of 20 cycles and peak negative pressure up to $300 \mathrm{kPa}$ at $1 \mathrm{MHz}$ in absence of Sonovue microbubbles did not vary for the total exposure duration. This curve demonstrates that no influence of ultrasound alone is observed on the cell membrane potential.

The influence of ultrasound in combination with microbubbles on the cell membrane potential is given in Fig. 5. Using acoustic waveforms of $200 \mathrm{kPa}, 1 \mathrm{MHz}, 10$ cycles and repeated every $100 \mu \mathrm{s}$, we measured a clear membrane hyperpolarization, which showed to be synchronized to the transmission of ultrasound waves. Moreover, the video observations revealed that only cells in a direct contact with the microbubbles are affected. The membrane remains hyperpolarized as long as the ultrasound is transmitted and the microbubble in contact with the cell membrane. Therefore a direct contact between the microbubble and the 
cell is essential and plays a key role in modulating the hyperpolarization amplitude. The variations of the membrane potential extended up to $25 \pm 1.4 \mathrm{mV}$ ( $\mathrm{n}=6$ cells). We should mention that the selected ultrasound conditions showed in a separate measurements increased uptake of a plasmid DNA. However to induce a membrane hyperpolarization, a pressure threshold is required. For acoustic pressures below $150 \mathrm{kPa}$, ultrasound and microbubbles did not affect the cell membrane potential.

Since a direct contact between the microbubbles and the cell is necessary, we assumed that the membrane hyperpolarization is caused by a mechanical stress induced by the microbubble oscillations. We have tested this hypothesis by applying manually a mechanical stress using a glass rod pressed against the cell while recording the membrane potential (Fig. 6a). We appreciate in this case a similar hyperpolarization phenomena of the membrane with amplitudes up to $-17 \mathrm{mV} \pm 1.9 \mathrm{mV}$ ( $\mathrm{n}=5$ cells) following the application of mechanical pressure on the cell as displayed in Fig. 6b. The hyperpolarization was repeatedly observed when the mechanical pressure was applied and the membrane potential returns to its resting value when the pressure is released.

We mention finally that the difference in the noise level between curves given in Figs. 4-6 can likely be attributed to the activity of the peristaltic pump which was continuously running and perfusing microbubbles to the explored medium, generating thus additional noise level.

\section{Effects of US and microbubbles on cells viability:}

The experimental conditions of the patch clamp measurements have been reproduced to investigate their influence on the cell viability. The results are summarized in Fig. 7, which shows the percentage of viable cells when exposed to different experimental conditions. Using ultrasound alone, Sonovue microbubbles alone or their association did not show any 
effect on cell viability using acoustic pressures up to $300 \mathrm{kPa}$ with no significant difference compared to the control. This figure demonstrates that the cells remain viable at these interrogation settings.

\section{Conclusions}

Besides diagnostic applications, gaseous microbubbles in combination with ultrasound waves have demonstrated today selective therapeutic benefits. Using ultrasound waves, microbubbles are capable to home to specific cellular targets through ultrasound focusing and modify by that the electrophysiological properties of cells in correlation with transmembrane exchange. While ultrasound alone and microbubbles alone have no detectable effects, a hyperpolarization of the membrane was measured when ultrasound waves are associated to microbubbles. The hyperpolarization has shown to be reversible and reproducible and can be associated with cell membrane deformation under the mechanical influence of microbubble oscillations. These variations induced by microbubbles and ultrasound observed in the membrane potential are similar to those induced by a mechanical pressure applied locally to the cell. This finding suggests strongly that mechanical oscillations of the cell membrane by microbubbles in a form of a "cellular massage" are sufficient to produce this effect. The hyperpolarization cannot be due to the formation of aspecific pores, which would tend to depolarize the cell, as described by (Deng et al. 2004). It is more likely that this interaction involves the activation of stretch sensitive ionic channels (Yang and Sachs 1990). Such activation can explain a decrease of transepithelial resistance since the opening of such ionic channels would increase cell permeability. However our results do not hint that membrane 
hyperpolarization is the cause of cell membrane permeabilization. However it might be possible that the hyperpolarisation of the membrane can attract charged molecules such as DNA and amplify their uptake by the cell and can be a promoter and or a consequence of the uptake mechanism.

However, the hyperpolarization does not seem to be the only consequence of microbubble oscillations since other effects such as potential depolarization might be triggered but still need to be elucidated. Therefore revealing the total action of ultrasound and microbubbles on the cell electrophysiological properties is a necessary step towards understanding mechanisms of cell membrane permeabilization with ultrasound and contrast microbubbles. 


\section{REFERENCES}

Christiansen JP, French BA, Klibanov AL, Kaul S, Lindner JR. Targeted tissue transfection with ultrasound destruction of plasmid-bearing cationic microbubbles. Ultrasound Med Biol 2003;29:1759-1767.

Deng CX, Sieling F, Pan H, Cui J. Ultrasound-induced cell membrane porosity. Ultrasound Med Biol 2004;30:519-526.

Goldberg B, Raichlen J, Forsberg F. Ultrasound Contrast Agents: Basic Principles and Clinical Applications., Dunitz M, Ed., 2nd ed, 2001.

Hamill OP, Marty A, Neher E, Sakmann B, Sigworth FJ. Improved patch-clamp techniques for high-resolution current recording from cells and cell-free membrane patches. Pflugers Arch 1981;391:85-100.

Marmottant P, Hilgenfeldt S. Controlled vesicle deformation and lysis by single oscillating bubbles. Nature 2003;423:153-156.

Mosmann T. Rapid colorimetric assay for cellular growth and survival: application to proliferation and cytotoxicity assays. J Immunol Methods 1983;65:55-63.

Neher E, Sakmann B. Single-channel currents recorded from membrane of denervated frog muscle fibres. Nature 1976;260:799-802.

Prentice P, Cuschieri A, Dholokia K, Prausnitz M, Campbell P. Membrane disruption by optically controlled microbubble cavitation. Nature Physics 2005;1:107-110.

Schneider M, Arditi M, Barrau MB et al. BR1: a new ultrasonographic contrast agent based on sulfur hexafluoride- filled microbubbles. Invest Radiol 1995;30:451-457.

Soule HD, Vazguez J, Long A, Albert S, Brennan M. A human cell line from a pleural effusion derived from a breast carcinoma. J Natl Cancer Inst 1973;51:1409-1416.

Tachibana K, Uchida T, Ogawa K, Yamashita N, Tamura K. Induction of cell-membrane porosity by ultrasound. Lancet 1999;353:1409.

Taniyama Y, Tachibana K, Hiraoka K et al. Local delivery of plasmid DNA into rat carotid artery using ultrasound. Circulation 2002;105:1233-1239.

Unger EC, Hersh E, Vannan M, Matsunaga TO, McCreery T. Local drug and gene delivery through microbubbles. Prog Cardiovasc Dis 2001;44:45-54.

Unger EC, Matsunaga TO, McCreery T et al. Therapeutic applications of microbubbles. Eur J Radiol 2002;42:160-168.

van Wamel A, Bouakaz A, Bernard B, ten Cate F, de Jong N. Radionuclide tumour therapy with ultrasound contrast microbubbles. Ultrasonics 2004;42:903-906.

Yang XC, Sachs F. Characterization of stretch-activated ion channels in Xenopus oocytes. J Physiol 1990;431:103-122. 


\section{LEGENDS}

Fig. 1. Schematic drawing of the patch clamp technique; a. seal between the glass pipette and the cell membrane is created, b. Control of the intracellular medium; c. Recordings of the membrane potential.

Fig. 2. Acoustical and electrophysiological experimental setup.

Fig. 3. Optical image of a patched cell and a Sonovue microbubbles in its close vicinity.

Fig. 4. Cell membrane potential of a patched cell with ultrasound alone $(1 \mathrm{MHz}, 200 \mathrm{kPa}$, pulse length $10 \mu \mathrm{s}$, repetition time $100 \mu \mathrm{s})$.

Fig. 5. Cell membrane potential of a patched cell with ultrasound (1 MHz, $200 \mathrm{kPa}$, pulse length $10 \mu$ s, repetition time $100 \mu$ s) and Sonovue microbubbles.

Fig. 6. a. Optical image of a glass rod pressing on a patched cell, b. Membrane potential of the patched cell under the rod mechanical pressure.

Fig. 7. Cell viability with different ultrasound conditions: US1=1 MHz, $300 \mathrm{kPa}$, pulse length $10 \mu \mathrm{s}$, repetition time $100 \mu \mathrm{s}, \mathrm{US} 2=1 \mathrm{MHz}, 200 \mathrm{kPa}$, pulse length $10 \mu \mathrm{s}$, repetition time $100 \mu \mathrm{s}$. 